\title{
ARTIGOS
}

Avaliado pelo processo double-blind review. Editora Científica convidada: Priscila Rezende da Costa

Versão traduzida | DOI: http://dx.doi.org/10.1590/So034-759020210607x

\section{ESTUDO SOBRE O COMPORTAMENTO DE USO DO LINE TODAY EM TAIWAN BASEADO NO MODELO UTAUT2}

\author{
A study of the use behavior of LINE TODAY in taiwan based on the UTAUT2 model \\ Un estudio sobre el comportamiento de uso de LINE TODAY en Taiwán basado en el modelo UTAUT2
}

Lisa Y. Chen ${ }^{1}$ | lisachen@isu.edu.tw | ORCID: 0000-0002-5418-6782

Yi-Jhen Chen ${ }^{1}$ | fanny3803003q@gmail.com | ORCID: 0000-0002-7953-1247

${ }^{1}$ I-Shou University, Departament of Information Management, Kaohsiung, Taiwan.

\section{RESUMO}

À medida que os negócios baseados em tecnologia móvel têm crescido e mudado o hábito de leitura de notícias das pessoas, o uso de plataformas móveis de notícias tem se tornado uma nova tendência. Devido a essa mudança, a taxa de utilização da plataforma de notícias Line Today de Taiwan foi a mais alta do mundo em 2018. 0 estudo utiliza o Modelo Estendido ao Consumo da Teoria Unificada da Aceitação e Uso de Tecnologia (Utaut2) para explorar as intenções comportamentais e o comportamento de uso do Line Today em Taiwan. Foi coletado um total de 199 amostras válidas. Os resultados mostram que a expectativa de desempenho, a expectativa de esforço, a influência social, as condições facilitadoras e o hábito afetam positivamente as intenções de comportamento. As intenções de comportamento e o hábito afetam positivamente o comportamento de uso. Os resultados oferecem ao Line Today e seus similares uma estratégia de aprimoramento, bem como referências para desenvolvimento e pesquisas futuras sobre plataformas móveis de notícias.

PALAVRAS-CHAVE | Plataforma móvel de notícias, intenção comportamental, comportamento de uso, Line Today, Utaut2.

\section{ABSTRACT}

As mobile business has flourished and people's news-reading habits have changed, the use of mobile news platforms has become a new trend. Due to this change, the use rate of LINE TODAY news from Taiwan became the world's highest in 2018. The study uses the Unified Theory of Acceptance and Use of Technology 2 (UTAUT2) model to explore behavioral intentions and the use behavior of LINE TODAY in Taiwan. A total of 199 valid samples were collected. Results show that performance expectancy, effort expectancy, social influence, facilitating conditions, and habit have positive impacts on behavior intentions. Behavior intentions and habit have positive impacts on use behavior. Results provide LINE TODAY and its peers with an improvement strategy and a reference for future development and research into mobile news platforms.

KEYWORDS I Mobile news platform, behavioral intention, use behavior, LINE TODAY, UTAUT2.

\section{RESUMEN}

A medida que los negocios basados en tecnología móvil han prosperado y cambiado los hábitos de lectura de noticias de las personas, el uso de plataformas móviles de noticias se ha convertido en una nueva tendencia. Debido a este cambio, la tasa de uso de la plataforma de noticias LINE TODAY en Taiwán fue la más alta del mundo en 2018. El estudio utiliza el modelo de teoría unificada de la aceptación y uso de tecnología 2 (UTAUT2) para explorar la intención conductual y el comportamiento de uso de LINE TODAY en Taiwán. Se recogió un total de 199 muestras válidas. Los resultados muestran que la expectativa de desempeño, expectativa de esfuerzo, influencia social, las condiciones facilitadoras y el hábito tienen impactos positivos en la intención conductual. A su vez, la intención conductual y el hábito tienen impactos positivos en el comportamiento de uso. Los resultados proporcionan a LINE TODAY y sus pares una estrategia de mejora, así como referencias para desarrollo e investigación futuros sobre las nuevas plataformas móviles de noticias.

PALABRAS CLAVE I Plataforma móvil de noticias, intención conductual, comportamiento de uso, LINE TODAY, UTAUT2. 


\section{INTRODUÇÃO}

Com o rápido desenvolvimento do comércio móvel, a tecnologia da informação (TI) está silenciosamente mudando os hábitos das pessoas no mundo moderno. 0 padrão de comportamento dos consumidores também tem mudado de "móvel como prioridade" para "somente móvel”, o que não apenas altera as relações de competição no âmbito das mídias, mas também dá origem a uma onda de necessidades de serviços (Market Intelligence \& Consulting Institute [MIC], 2016). À medida que as necessidades de socialização, informações, games ou compras têm crescido, as mídias sociais têm se tornado uma importante estratégia de marketing para as empresas e uma necessidade no cotidiano das pessoas (Appel, Grewal, Hadi, \& Stephen, 2019).

A tecnologia digital tem modificado os hábitos de leitura de notícias das pessoas. 0 acesso dos leitores às notícias passou dos jornais tradicionais às plataformas sociais por meio de dispositivos móveis. A diversificação de serviços de notícias com base em tecnologia móvel permite aos usuários acessar conteúdos de notícias de maneira fácil, a qualquer hora e em qualquer lugar em dispositivos móveis, visitando sites de notícias ou fazendo login em mídias sociais (Lee, Lindsey, \& Kim, 2017). À medida que as plataformas de mídia mudaram o comportamento dos usuários on-line, a forma de ir diretamente a sites de notícias para navegar pelas principais reportagens, ou de utilizar o Google para buscar os principais fatos noticiados, tem mudado dramaticamente (China Times, 2015).

Nos últimos anos, o Line tem se comprometido com a oferta de serviços diversificados centrados nas necessidades do usuário, incluindo notícias, games, mapas, pagamentos e e-commerce em cinco áreas principais (Business Next, 2016; China Times, 2017b). O Today é uma plataforma móvel global de notícias oferecida pelo Line, com conteúdo premium de veículos parceiros, permitindo aos usuários navegar, armazenar, deixar mensagens ou compartilhar notícias no Line Today a qualquer hora. Os usuários podem ir diretamente a ele para ler notícias, passando à esquerda e à direita para a página de notícias, e para cima e para baixo para navegar por tópicos de conteúdos ricos de notícias, tais como temas de interesse, entretenimento e vida (Line Today, 2017). Em 2018, o número de usuários ativos da plataforma por mês em Taiwan estabeleceu um novo recorde, de 21 milhões, com reportagens e textos do Line, levando o consumo de notícias do Line Today a ocupar o primeiro lugar no mundo (Business Next, 2018).

De acordo com o ETtoday (2018), os leitores taiwaneses dependem fortemente das mídias sociais, especialmente do Facebook e do Line, $56 \%$ deles recebendo suas notícias desses canais, mas essas mídias sociais têm relativamente pouca responsabilidade social. O Digital News Report (2018) apontou que fake news produzidas na China no ano passado espalharam-se em Taiwan e na mídia taiwanesa, sendo esta profundamente influenciada pelas mídias sociais, o que contribuiu para a disseminação dessas fake news. Está claro que as plataformas de mídias sociais podem gerar dados de maneira fácil e rápida, e disseminar mensagens instantâneas, tornando mais e mais pessoas vulneráveis a informações intencionalmente enganosas. Em comparação com as fontes tradicionais de notícias, no entanto, a liberdade que as mídias sociais proporcionam torna a credibilidade do conteúdo disseminado nas plataformas questionável (Meel \& Vishwakarma, 2019).

Os principais usuários do Line Today em Taiwan têm mais de 25 anos de idade, e a proporção de homens é significativamente maior do que a de mulheres. O Line Today é conveniente para os usuários buscarem e lerem notícias no contexto dos chats. A maioria das usuárias costuma dar curtidas em vez de deixar mensagens. Mercados como a Indonésia e a Tailândia gostam de ver conteúdos originais de comunidades. A vantagem do Line Today é que a plataforma possui uma base grande de usuários que as outras plataformas não possuem (ChinaTimes, 2017). 
A plataforma deve satisfazer não apenas a oferta de conteúdo instantâneo, mas também necessita ser personalizável para as pessoas. Em comparação com outras plataformas de notícias, o Line Today tem modificado os hábitos dos usuários que leem notícias e o modo como eles utilizam o site. Em Taiwan, o Line Today possui um grande número de usuários, o que facilita a realização de pesquisas on-line, e é importante observar os fatores que levam os usuários a adotar esse canal. Assim, o objetivo deste estudo é explorar o comportamento de uso do telefone celular para a leitura de notícias em substituição aos meios tradicionais, tomando o Line Today como um estudo de caso. 0 estudo baseia-se no Modelo Estendido ao Consumo da Teoria Unificada da Aceitação e Uso da Tecnologia (Utaut2), com bom poder explicativo para entender as intenções comportamentais e 0 comportamento de uso do Line Today.

Este estudo divide-se em cinco partes, da seguinte forma: a primeira parte é uma introdução, que descreve os antecedentes, o objetivo e a importância deste estudo. A segunda parte discute o significado da revisão da literatura relevante. A terceira parte apresenta a estrutura conceitual, juntamente com as hipóteses e os métodos estatísticos utilizados. A quarta parte resume os dados coletados, os resultados da análise dos dados e os achados. A última parte apresenta uma conclusão significativa, incluindo as contribuições, recomendações e implicações deste estudo, bem como direcionamentos futuros.

\section{REVISÃO DA LITERATURA}

Mudanças rápidas na tecnologia digital têm possibilitado que novas tecnologias continuem a evoluir em poucos anos, levando a alterações nos padrões de consumo e ao desenvolvimento das mídias sociais, e redefinindo o conceito de comunicação de notícias. As plataformas sociais não apenas introduzem o consumo de imagens, vídeos e música, mas também ajudam os consumidores a julgar a autenticidade das notícias ao permitir comentários de usuários de redes sociais (Tavis, 2012). A popularidade dos dispositivos móveis, no que tange às plataformas sociais, representou uma grande disrupção na tradição da indústria de notícias. Mais de 50\% de todos os usuários de dispositivos móveis em Taiwan utilizam a internet como sua principal fonte de notícias, incluindo redes sociais. A tendência do uso de plataformas sociais móveis por serviços de notícias deve-se principalmente à ascensão dos sites de redes sociais.

No início, os portais de notícias permitiam apenas que os usuários recebessem as notícias em uma direção, até que surgiu o Yahoo! Kimo para notícias, e, com ele, a função de mensagens de mão dupla. No entanto, desde seu lançamento, em 2016, o Line Today tem se tornado um companheiro inseparável do povo taiwanês. 0 Line Today difere do Yahoo! Kimo principalmente por seus aplicativos para celular, enquanto o Yahoo! Kimo para notícias é um produto voltado para computador pessoal (PC). O Line Today criou um portal de conteúdo que permite aos leitores assistirem a notícias com um clique. Além disso, oferece conteúdo diversificado e integrado. Depois que o Line passou a oferecer seu serviço Line Points, as pessoas passaram a assistir a notícias no Line Today em seus PCs, e desenvolveram o hábito de fazê-lo várias vezes ao dia (Global Views, 2018). Muitos taiwaneses usam o Line Today para assistir a notícias, e a plataforma de mídia está crescendo tão rapidamente que passa a merecer atenção devido ao seu desenvolvimento.

\section{0 modelo de aceitação da tecnologia e teorias correlatas}

Muitos estudos exploraram a adoção de diferentes modelos no tocante à aceitação de novas tecnologias pelo usuário, tais como a Teoria da Ação Racional (TRA), o Modelo de Aceitação da Tecnologia (TAM), a Teoria Unificada 
da Aceitação e Uso da Tecnologia (Utaut) e o Modelo Estendido ao Consumo da Teoria Unificada da Aceitação e Uso da Tecnologia (Utaut2). Davis (1989) sugeriu que TAM era a ferramenta mais amplamente utilizada e aceita em vários campos de pesquisa, principalmente com base na TRA de Fishbein e Ajzen (1975), que tenta prever e entender o comportamento humano.

A TAM é utilizada principalmente para explicar a adoção de sistemas de informação (SI) por funcionários de uma organização e para prever o comportamento dos usuários. A utilidade e facilidade de uso percebidas são as duas variáveis de crença importantes desse modelo, e afetam a atitude em relação ao uso da tecnologia, a qual, por sua vez, afeta a intenção do usuário de utilizá-la e seu comportamento efetivo. No entanto, o modelo concentra-se apenas na perspectiva dessas duas variáveis, e o impacto de outras variáveis externas importantes pode ser ignorado. Os estudos também mostram uma precisão de apenas $40 \%$ na previsão do uso de um sistema pelo usuário, embora a TAM possua uma fundamentação teórica simples e clara. Assim, alguns estudos alcançaram melhorias em relação ao modelo e ofereceram sugestões.

Venkatesh, Morris, Davis e Davis (2003) realizaram melhorias e ofereceram sugestões com base nas limitações dos modelos anteriores, e desenvolveram o modelo Utaut. Seus estudos confirmaram que os fatores de identificação do modelo Utaut afetam diretamente as intenções de comportamento e podem prever o comportamento de uso efetivo. Embora, em pesquisas sobre o comportamento de aceitação de novas tecnologias, o modelo Utaut apresente um bom poder explicativo, em alguns estudos recentes, os resultados e a capacidade de explicar a aceitação da tecnologia pelos indivíduos não são exatamente os mesmos do modelo Utaut (Chao, 2019).

O modelo Utaut geralmente se concentra em explicar a aceitação de um novo sistema por funcionários de uma organização, mas considera apenas a motivação externa para a utilização da Tl, deixando de explicar a adoção não organizacional de novas tecnologias e de considerar as motivações internas dos usuários. Assim, Venkatesh, Thong e Xu (2012) propuseram uma extensão ao modelo Utaut, a saber, a Utaut2, que se concentra nas perspectivas individuais com relação à adoção de tecnologias. Eles mudaram o contexto, passando da organização para o consumidor, e introduziram novos construtos e relações no modelo. 0 modelo Utaut2 revisou os modelos acima, buscando atender à necessidade de incluir preditores significativos que possam ser utilizados em vários contextos de adoção da tecnologia móvel pelo usuário.

No entanto, estudos recentes, como os realizados por Dwivedi, Rana, Jeyaraj, Clement e Williams (2019), têm argumentado que o modelo Utaut infere teoricamente relações que podem não ser aplicáveis a todos os contextos, não considera algumas relações potencialmente importantes e exclui alguns construtos que podem ser cruciais para explicar a aceitação e o uso da TI/SI. Assim, Shiferaw e Mehari (2019) estenderam a Utaut e utilizaram determinantes específicas de contexto em suas pesquisas, a fim de melhorar sua capacidade preditiva.

Diferentemente de outros estudos que utilizam principalmente a Utaut ou a Utaut2 como base teórica, Hallikainen, Alamäki e Laukkanen (2019) consideraram que as pesquisas existentes geralmente se concentram no comportamento on-line, especialmente na adoção de tecnologia móvel, acreditando que a adoção da tecnologia seja um comportamento inovador. Dada essa limitação, eles ampliaram a teoria do lead user como referencial teórico, com base na teoria da inovatividade pessoal, e concentraram-se no comportamento inovador de lead users de serviços móveis para empresas. No entanto, sua amostra era composta por executivos, gerentes e tomadores de decisão, portanto as opiniões podem ser diferentes das visões dos consumidores.

Entre todas as teorias e modelos que se concentram em perspectivas individuais e as utilizam para prever a aceitação e o uso de tecnologias, a Utaut2 vem gradualmente se tornando a base teórica da maioria dos estudos, 
e possui um elevado poder explicativo. Como resultado, este estudo busca verificar se esse modelo tem bom poder explicativo, tendo por objetivo explorar as intenções comportamentais em relação ao Line Today, bem como o comportamento de uso, e a Utautz é adequada para este estudo. As seções a seguir discutem com mais detalhe a Utaut e a Utaut2, respectivamente.

\section{0 modelo Utaut}

Venkatesh et al. (2003) examinaram estudos relevantes ao longo dos anos e concluíram que a teoria empírica possui diferentes capacidades explicativas em diferentes categorias. Eles compararam os oito modelos de aceitação de tecnologia anteriores, discutiram suas diferenças, realizaram a validação cruzada dos dados e propuseram uma teoria unificada de aceitação e uso da tecnologia (Utaut). 0 modelo Utaut inclui os seguintes: 1. Teoria do Comportamento Planejado (TPB); 2. TRA; 3. TAM; 4. Modelo de Utilização de PC (MPCU); 5. Combinação entre TAM e TPB (C-TAM-TPB); 8. Teoria Cognitiva Social (SCT). Eles integraram os modelos anteriores sobre aceitação de tecnologia de modo a oferecer um modelo melhor e mais completo de como a Tl é utilizada. 0 poder explicativo do comportamento do usuário chega a 70\%, muito acima dos outros modelos teóricos anteriores, o que indica que suas variáveis de ajuste oferecem um poder explicativo mais completo sobre o comportamento do usuário (Cao \& Niu, 2019; Chopdar, Korfiatis, Sivakumar, \& Lytras, 2018).

No modelo Utaut mostrado na Figura 1, os argumentos propostos na literatura pertinente foram integrados em quatro dimensões: expectativa de desempenho (ED), expectativa de esforço (EE), influência social (IS) e condições facilitadoras (CF). Além disso, quatro variáveis moderadoras foram adicionadas: gênero, idade, experiência e voluntariedade do uso. 0 modelo Utaut defende que a intenção de comportamento afeta o comportamento do usuário. A intenção de comportamento é afetada principalmente pela ED, pela EE e pela IS, enquanto os dois fatores que afetam diretamente o comportamento do usuário são as CF e a intenção de comportamento. A supracitada influência será afetada por quatro variáveis de interferência: gênero, idade, experiência e voluntariedade de uso.

\section{Figura 1. Modelo UTAUT}

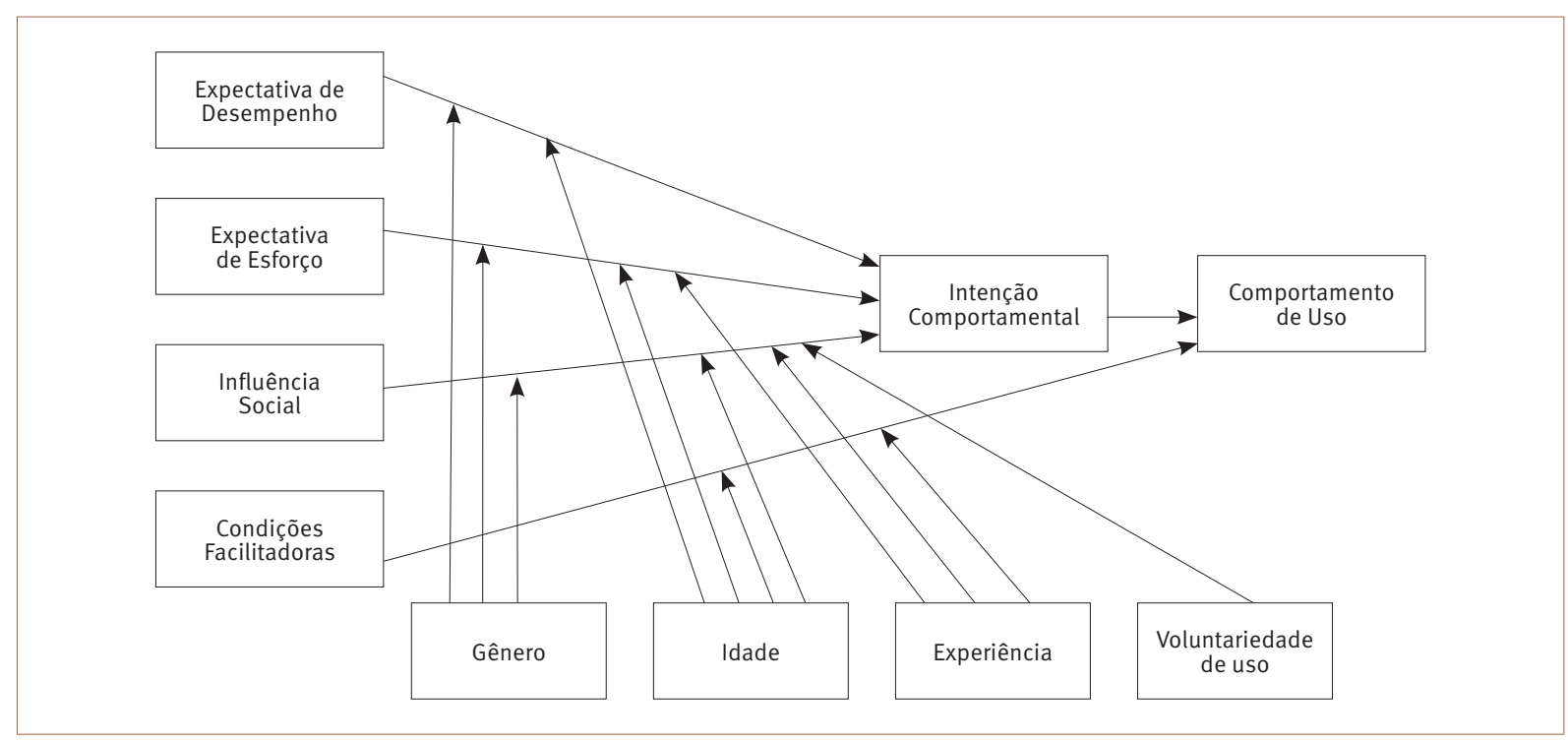

Fonte: Venkatesh et al., 2003. 


\section{O modelo Utaut2}

Após examinar as pesquisas empíricas, Venkatesh et al. (2012) revisaram e estenderam o modelo Utaut existente, adicionando à estrutura teórica três novos fatores: motivação hedônica, valor de preço e hábito, conforme mostrado na Figura 2. Em comparação com a Utaut, a Utaut2 considera as perspectivas individuais na adoção de tecnologias e tem maior poder explicativo, pois reduz as variáveis de ajuste a gênero, idade e experiência. 0 poder explicativo da intenção de comportamento na Utaut2 aumentou de $56 \%$ para $74 \%$. O poder explicativo do uso de tecnologias aumentou de $40 \%$ para $52 \%$.

\section{Figura 2. Modelo UTAUT2}

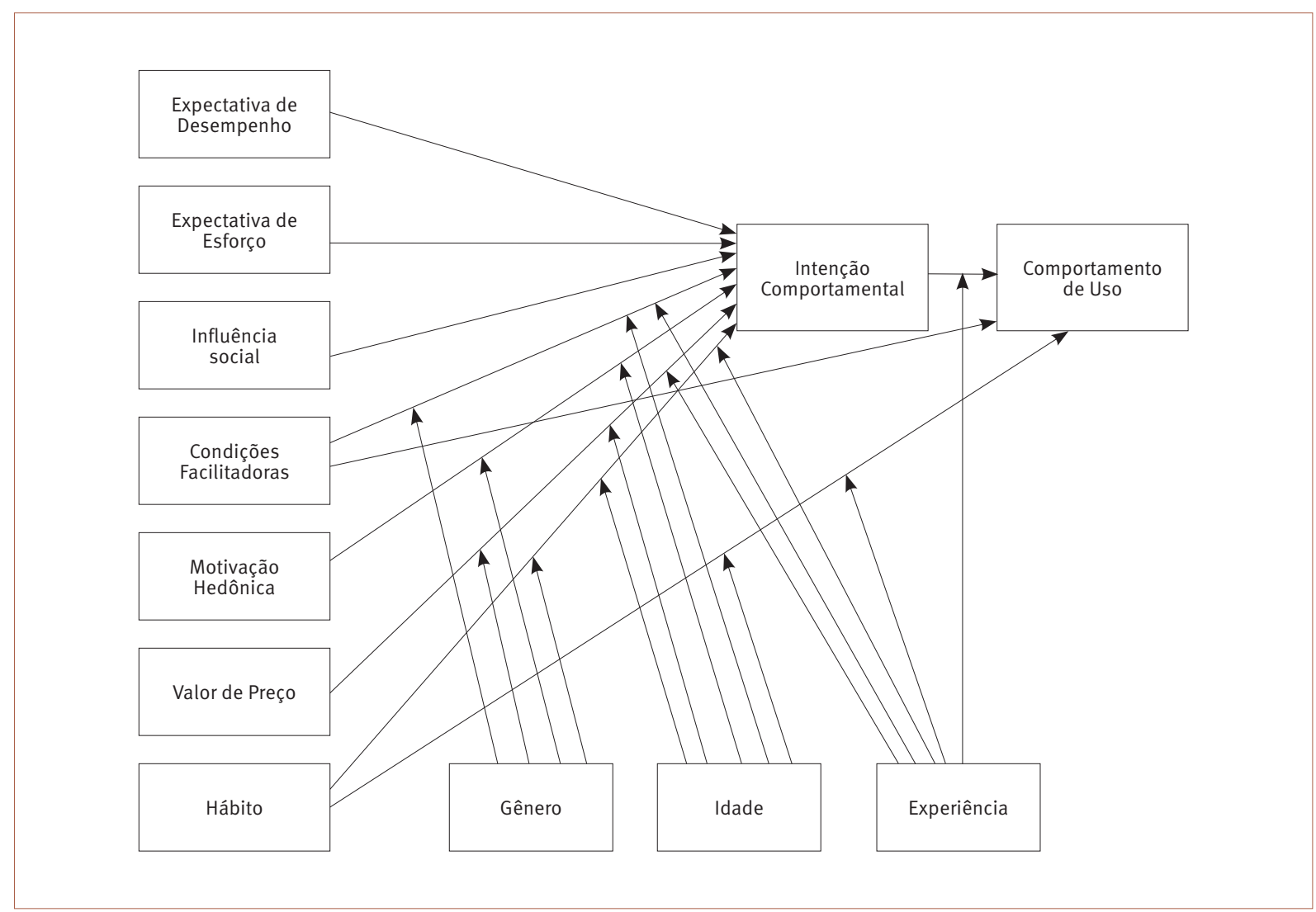

Fonte: Venkatesh et al., 2012

Como o modelo da Utaut foi inicialmente discutido do ponto de vista dos usuários de sistemas empresariais, Venkatesh et al. (2012) acreditavam que ele poderia ser amplamente utilizado com diferentes tecnologias. Portanto, além de adicionar novas variáveis, a Utaut2 também introduz algumas mudanças na relação entre as variáveis originais, por exemplo: as condições facilitadoras não afetam apenas o comportamento de uso, mas também a intenção comportamental. As definições de ED, EE, IS e CF também foram um pouco revisadas. Quanto ao aspecto das variáveis de ajuste, a interferência voluntária não é mais discutida neste modelo.

Com base na Utaut2, muitos pesquisadores conduziram avaliações e estudos correlatos para verificar o comportamento de uso e a intenção de uso, e todas essas pesquisas apresentam bom poder explicativo (Alalwan, 
2020; Herrero, Martín, \& Salmones, 2017; Ramírez-Correa, Rondán-Cataluña, Arenas-Gaitán, \& Martín-Velicia, 2019; Tamilmani, Rana, Prakasam, \& Dwivedi, 2019). Acredita-se que o uso da Utaut2 para investigar o comportamento de adoção individual esteja mais próximo do ambiente atual do usuário. Assim, este estudo analisou o modelo Utaut2 como referencial básico para investigar o comportamento de uso e os fatores associados ao uso do Line Today. A motivação hedônica e o valor de preço não serão incluídos neste estudo, pois o Line Today é uma plataforma móvel de notícias gratuita, sem implicações de custo, e não possui motivação hedônica. As hipóteses do estudo são desenvolvidas de modo a explorar as relações entre as variáveis, com base no referencial conceitual.

\section{0 efeito da expectativa de desempenho, da expectativa de esforço, da influência social, das condições facilitadoras e do hábito sobre a intenção comportamental}

Estudos recentes sobre games de redes sociais móveis (Baabdullah, 2018), aulas colaborativas suportadas por computador (Ali, Nair, \& Hussain, 2016) e plataformas de streaming de vídeo (Weng \& Huang, 2017) concluíram que a ED, a EE, a IS e as CF apresentam um impacto positivo sobre a intenção comportamental de utilizar novas tecnologias. Os hábitos também afetam a intenção comportamental de utilizar novas tecnologias (Ramírez-Correa et al., 2019). Este estudo conclui que quanto maiores forem a ED, a EE e as CF para o uso do Line Today, maior será a intenção de utilizá-lo. As opiniões dos outros e o hábito também afetam a intenção de utilizar o Line Today. As hipóteses são as seguintes:

H1: A expectativa de desempenho afeta positivamente a intenção comportamental de utilizar o Line Today.

H2: A expectativa de esforço afeta positivamente a intenção comportamental de utilizar o Line Today.

H3: A influência social afeta positivamente a intenção comportamental de utilizar o Line Today.

H4: As condições facilitadoras afetam positivamente a intenção comportamental de utilizar o Line Today.

$\mathrm{H}_{5}$ : O hábito afeta positivamente a intenção comportamental de utilizar o Line Today.

\section{O efeito da intenção comportamental, das condições facilitadoras e do hábito sobre o comportamento de uso}

Venkatesh et al. (2012) concluíram que tanto as intenções comportamentais quanto as condições facilitadoras apresentam impactos diretos sobre o comportamento de uso em relação a tecnologias. Com base no ambiente de tecnologia dos usuários, após incorporar a variável "hábito" ao modelo Utaut2, eles constataram que o hábito afeta o comportamento de uso quando novas tecnologias estão sendo adotadas. Enquanto isso, estudos relacionados sobre games on-line móveis e plataformas de streaming de vídeo também apoiam a correlação (Ramírez-Correa et al., 2019; Weng \& Huang, 2017). Com base no que foi dito, este estudo infere que quanto maiores a intenção comportamental, as CF e o hábito de utilizar o Line Today, maior o comportamento de uso do Line Today. As hipóteses são as seguintes:

H6: A intenção comportamental afeta positivamente o comportamento de uso com relação ao uso do Line Today.

H7: As condições facilitadoras afetam positivamente o comportamento de uso com relação ao uso do Line Today.

H8: O hábito afeta positivamente o comportamento de uso com relação ao uso do Line Today. 


\section{MÉTODO DE PESQUISA}

Com base nas motivações e objetivos de pesquisa referidos acima, este estudo estabeleceu o modelo e hipóteses de pesquisa e as definições operacionais por meio do estudo e da discussão da literatura relevante. Considerando as restrições econômicas, de tempo e de recursos, além de outros fatores relacionados à coleta de dados, o estudo adotou um método de amostragem não aleatório e por conveniência. Assim, os respondentes não estão excessivamente concentrados em um grupo específico, mas espalhados entre usuários em diferentes níveis. Um questionário presencial e um questionário on-line (www.surveycake.com) foram simultaneamente distribuídos aos respondentes, que foram convidados a preenchê-los utilizando um código QR.

\section{Modelo de pesquisa e definição operacional}

O estudo elaborou um framework de pesquisa (conforme mostrado na Figura 3), com base na Utaut2, que explica o comportamento de uso do Line Today. Neste estudo, a definição de expectativa de desempenho refere-se a quanto os usuários acreditam que o Line Today pode tornar sua visualização de notícias mais eficiente ou recompensadora. A expectativa de esforço refere-se à percepção do usuário sobre a simplicidade de operação do Line Today. A influência social refere-se a quanto os usuários sentem a influência de seus parentes e amigos sobre seu uso do Line Today. As condições facilitadoras referem-se a como os usuários acreditam que as tecnologias de software e hardware e recursos de equipamentos podem apoiar os indivíduos que utilizam o Line Today. $O$ hábito refere-se a quanto os usuários utilizam o Line Today, sendo afetado por hábitos e experiências de operação anteriores. A intenção comportamental é definida como as ações específicas ou orientações comportamentais futuras tomadas pelos usuários após utilizar o Line Today. 0 comportamento de uso refere-se ao tempo e à frequência com que o usuário efetivamente utiliza o Line Today.

\section{Figura 3. Modelo de pesquisa do estudo}

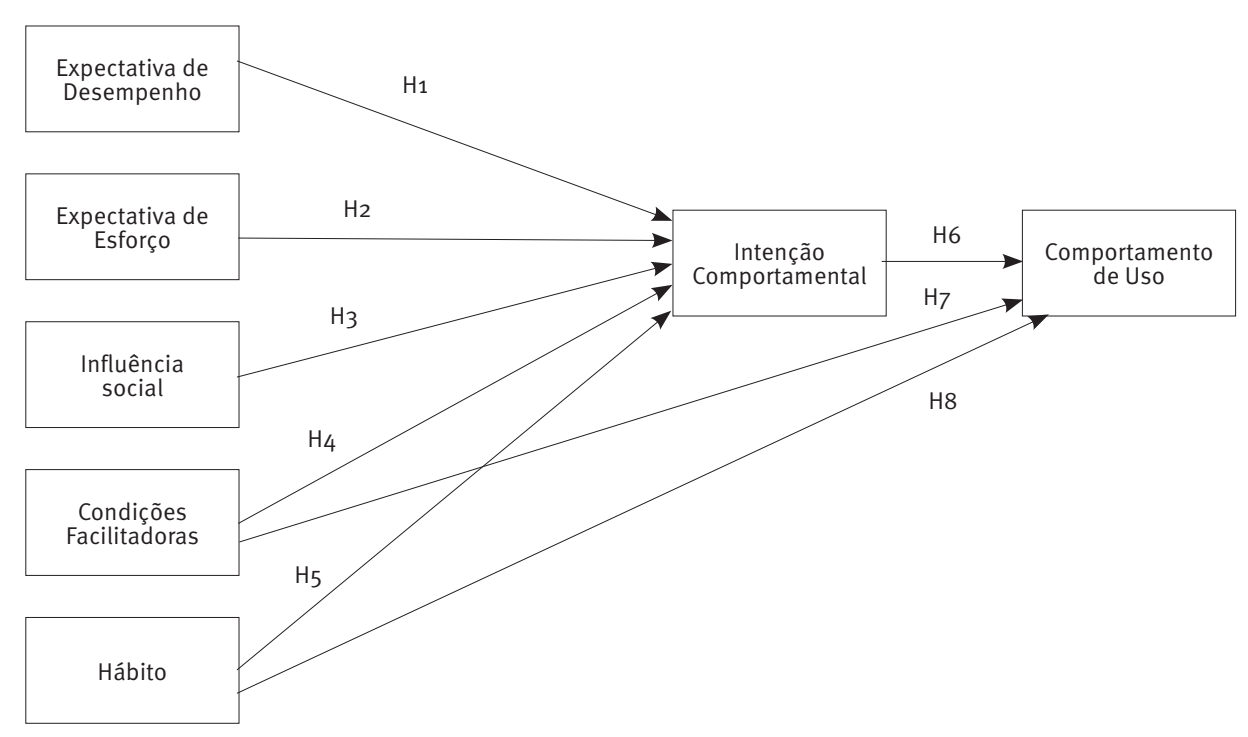




\section{Medição}

O estudo utiliza o método de pesquisa por questionário para coletar dados, e foram consultados especialistas a respeito de emendas ao questionário. A estrutura do questionário do estudo divide-se em três partes. No início do instrumento, perguntava-se aos respondentes se eles já haviam utilizado o Line Today. Se sim, eles continuavam a preencher o questionário. Se não, paravam de responder. Finalmente, a primeira e a terceira parte do instrumento foram medidas na escala nominal, enquanto a segunda parte do questionário adotou uma escala Likert de 5 pontos para medir o grau de aprovação do respondente.

As questões foram compiladas em uma escala contendo 27 questões de mensuração, em que 1 correspondia a "Discordo fortemente" e 5 correspondia a "Concordo fortemente". Os resultados desse questionário foram estatisticamente processados e analisados utilizando o software PASW® Statistics. Com base em uma amostra de tamanho pequeno, utilizou-se a regressão por mínimos quadrados parciais (PLS), com o SmartPLS 3.0, para modelagem por equações estruturais (SEM), a fim de avaliar o modelo de medição e o modelo de estrutura deste estudo.

\section{Amostra e coleta de dados}

Um questionário revisado baseou-se nos resultados do teste piloto. No teste, 60 amostras foram coletadas, sendo seis inválidas e 54 válidas, com um índice de coleta de $90 \%$. Os resultados do teste piloto mostraram que o coeficiente de confiabilidade estava acima de 0,8 para todas as variáveis e foi considerado aceitável (Nunnally, 1978). Foi coletado um total de 230 questionários, excluindo-se 31 por estarem incompletos ou porque os respondentes nunca haviam utilizado o Line Today. O número de questionários válidos (199 de 230) recebidos proporcionou uma taxa de retorno de 86,5\%, alcançando os critérios sugeridos por Harris (1985). De acordo com Chin (1998), o PLS pode ser adequado a dados de um número pequeno de amostras, sendo o requisito mínimo de 30 a 100 amostras. Além disso, o estudo utiliza a repetição de amostras por bootstrapping para testar se os coeficientes de caminho alcançam um nível significativo. Normalmente, 500 amostras bootstrap podem ser suficientes para obter estimativas de amostra. Neste estudo, o método de reamostragem por bootstrapping foi utilizado para gerar mil amostras bootstrap, a fim de melhorar os poderes computacionais para estimativa de parâmetro e inferência quando a amostragem é restrita pelo pequeno tamanho da amostra original. Assim, o número de amostras deste estudo está de acordo com o limite requerido de amostras para o método de PLS, sendo adequado para a análise de modelo estrutural.

\section{Resultados da análise de dados}

Utilizou-se primeiro a análise descritiva dos dados para sintetizar as características básicas dos dados da amostra. Em seguida, utilizou-se a análise de dados para confirmar a adequação dos itens da escala, que é também a base para medir a confiabilidade e validade gerais. Utilizou-se a análise fatorial para testar a validade dos construtos de um questionário. Uma vez que as variáveis observadas e os construtos latentes do modelo de pesquisa foram baseados na Utaut2, utilizou-se uma análise fatorial confirmatória para verificar a correlação entre os fatores da análise, a fim de obter a confiabilidade composta (CC), a variância média extraída (VME) e os coeficientes de correlação entre os construtos para obter a validade convergente e discriminante. Para avaliar a confiabilidade da medição, foram utilizadas correlações item-escala para examinar a validade de construto dos 
itens, com uma carga maior do que 0,5 e valores de a de Cronbach maiores do que 0,7. A CC também foi considerada para a avaliação da consistência interna na confiabilidade de construto por preservar as cargas padronizadas das variáveis observadas. Utilizou-se a validade discriminante, comparando-se a VME dos pares de construto à correlação quadrada entre eles. 0 modelo estrutural foi empregado estimando-se os caminhos entre os construtos do modelo. A significância dos coeficientes de caminho foi determinada utilizando estatísticas t calculadas por meio da técnica de bootstrapping.

\section{Análise das estatísticas descritivas}

A proporção de respondentes do sexo feminino foi maior do que a de respondentes do sexo masculino (58,3\% de mulheres e $41,7 \%$ de homens). A maioria dos respondentes (53,8\%) tinha entre 21 e 30 anos de idade, seguida por indivíduos de 20 anos de idade ou menos (47,7\%). 0 estudo investigou ainda o nível educacional, a ocupação e a renda média mensal. Os respondentes com nível universitário representavam $87,9 \%$ da amostra, seguidos por indivíduos com pós-graduação, representando 11,1\%. A renda média mensal de 83,9\% dos respondentes é inferior a NT\$20.000,00, 10,1\% possuindo renda média entre NT\$20.001,00 e NT\$30.000,00 (NT= Dólar Taiwanês). Com relação à ocupação, 83,4\% eram estudantes, seguidos por indivíduos que trabalham no setor de $\mathrm{TI}$, representando $4,5 \%$. Com relação ao padrão de uso, os resultados mostram que $73,9 \%$ dos respondentes utilizam o Line Today todos os dias por, no mínimo, 30 minutos, enquanto 24,6\% utilizam o Line Today menos do que um dia por semana. Com relação ao canal utilizado, $89,2 \%$ dos respondentes assistem ao Line Today em seus dispositivos móveis.

\section{Teste de confiabilidade e validade}

Foi realizada uma análise fatorial confirmatória utilizando PLS com o SmartPLS 3.0. Os resultados estatísticos são mostrados na Tabela 1. Para avaliar a confiabilidade e validade das variáveis observadas, foram empregadas as cargas de todos os itens da escala para testar a validade dos construtos, variando as correlações dos itens da escala entre 0,764 e 0,919, enquanto os valores da VME dos construtos ficaram entre 0,658 e 0,802. Com relação aos critérios, a validade convergente demonstrou que as cargas das variáveis observadas eram maiores do que o,7, e os valores de VME para cada construto eram maiores do que o padrão de 0,5. 0 teste t do valor estimado de cada item alcançou um nível significativo (p<o.001), considerado bastante aceitável. Conforme mostra a Tabela 1, os valores da CC dos construtos variavam entre 0,885 e 0,948, ficando acima do limite recomendado de 0,7 , indicando que os construtos deste estudo possuem consistência interna. Os valores do a de Cronbach foram maiores do que 0,8 , indicando que o modelo de medição possuía confiabilidade aceitável, conforme sugerido por Hair, Anderson, Tatham, \& Black (1998).

Para testar a validade discriminante, utilizou-se a raiz quadrada da VME dos construtos individuais como critério de julgamento. A partir da matriz dos coeficientes de correlação entre os construtos da Tabela 2, o coeficiente de correlação de cada construto deste estudo ficou entre o,612 e 0,838, e a raiz quadrada do valor da VME de cada construto foi maior do que a correlação entre dois construtos quaisquer, mostrando que o questionário elaborado para uso neste estudo possuía validade discriminante suficiente. 
ARTIGOS | ESTUDO SOBRE O COMPORTAMENTO DE USO DO LINE TODAY EM TAIWAN BASEADO NO MODELO UTAUT2

Lisa Y. Chen | Yi-Jhen Chen

Tabela 1. Confiabilidade e validade dos construtos

\begin{tabular}{|c|c|c|c|c|c|c|}
\hline Construtos & Itens & Cargas & Estatística t & $A$ & $\mathrm{CC}$ & VME \\
\hline \multirow{5}{*}{$\begin{array}{l}\text { Expectativa de Desempenho } \\
\text { (ED) }\end{array}$} & ED1 & 0,888 & 64,494 & \multirow{5}{*}{0,909} & \multirow{5}{*}{0,932} & \multirow{5}{*}{0,734} \\
\hline & $\mathrm{ED} 2$ & 0,856 & 45,468 & & & \\
\hline & $\mathrm{ED}_{3}$ & 0,841 & 33,173 & & & \\
\hline & ED4 & 0,852 & 39,465 & & & \\
\hline & $\mathrm{ED}_{5}$ & 0,845 & 34,532 & & & \\
\hline \multirow{5}{*}{ Expectativa de Esforço (EE) } & $\mathrm{EE} 1$ & 0,844 & 37,869 & \multirow{5}{*}{0,899} & \multirow{5}{*}{0,925} & \multirow{5}{*}{0,712} \\
\hline & $\mathrm{EE} 2$ & 0,849 & 32,730 & & & \\
\hline & $\mathrm{EE}_{3}$ & 0,824 & 27,028 & & & \\
\hline & $\mathrm{EE}_{4}$ & 0,867 & 34,429 & & & \\
\hline & $\mathrm{EE}_{5}$ & 0,834 & 33,207 & & & \\
\hline \multirow{4}{*}{ Influência Social (IS) } & IS1 & 0,894 & 49,772 & \multirow{4}{*}{0,918} & \multirow{4}{*}{0,942} & \multirow{4}{*}{0,802} \\
\hline & IS2 & 0,901 & 74,636 & & & \\
\hline & IS3 & 0,869 & 37,599 & & & \\
\hline & $1 S_{4}$ & 0,919 & 70,480 & & & \\
\hline \multirow{4}{*}{ Condições Facilitadoras (CF) } & $\mathrm{CF}_{1}$ & 0,791 & 21,255 & \multirow{4}{*}{0,827} & \multirow{4}{*}{0,885} & \multirow{4}{*}{0,658} \\
\hline & $\mathrm{CF}_{2}$ & 0,764 & 21,457 & & & \\
\hline & $\mathrm{CF}_{3}$ & 0,846 & 40,479 & & & \\
\hline & $\mathrm{CF}_{4}$ & 0,841 & 38,749 & & & \\
\hline \multirow{5}{*}{ Hábito (HA) } & $\mathrm{HA} 1$ & 0,912 & 61.920 & \multirow{5}{*}{0,931} & \multirow{5}{*}{0,948} & \multirow{5}{*}{0,784} \\
\hline & $\mathrm{HA} 2$ & 0,897 & 44,411 & & & \\
\hline & $\mathrm{HA}_{3}$ & 0,860 & 37,619 & & & \\
\hline & $\mathrm{HA}_{4}$ & 0,897 & 53,833 & & & \\
\hline & $\mathrm{HA}_{5}$ & 0,859 & 28,030 & & & \\
\hline \multirow{4}{*}{ Intenção Comportamental (IC) } & $\mathrm{IC}_{1}$ & 0,898 & 61,000 & \multirow{4}{*}{0,905} & \multirow{4}{*}{0,932} & \multirow{4}{*}{0,779} \\
\hline & $\mathrm{IC}_{2}$ & 0,870 & 49,133 & & & \\
\hline & $\mathrm{IC}_{3}$ & 0,882 & 45,827 & & & \\
\hline & $\mathrm{IC}_{4}$ & 0,879 & 54,284 & & & \\
\hline \multirow{2}{*}{$\begin{array}{l}\text { Comportamento do Usuário } \\
\text { (CDU) }\end{array}$} & $\mathrm{CDU}_{1}$ & 0,769 & 25,563 & \multirow{2}{*}{0,816} & \multirow{2}{*}{0,842} & \multirow{2}{*}{0,728} \\
\hline & $\mathrm{CDU}_{2}$ & 0.922 & 57.975 & & & \\
\hline
\end{tabular}

Tabela 2. Validade discriminante

\begin{tabular}{l|c|c|c|c|c|c|c}
\hline & ED & EE & IS & CF & HA & IC & CDU \\
\hline Expectativa de Desempenho (ED) & 0,857 & & & & & \\
\hline Expectativa de Esforço (EE) & 0,730 & $\mathbf{0 , 8 4 4}$ & & & & \\
\hline Influência Social (IS) & 0,612 & 0,619 & $\mathbf{0 , 8 9 6}$ & & & \\
\hline Condições Facilitadoras (CF) & 0,711 & 0,670 & 0,629 & $\mathbf{0 , 8 1 1}$ & & & \\
\hline Hábito (HA) & 0,748 & 0,698 & 0,624 & 0,739 & 0,885 & & \\
\hline Intenção Comportamental (IC) & 0,801 & 0,776 & 0,714 & 0,838 & 0,799 & 0,882 & \\
\hline Comportamento do Usuário (CDU) & 0,766 & 0,771 & 0,735 & 0,796 & 0,816 & 0,832 & $\mathbf{0 , 8 4 9}$ \\
\hline
\end{tabular}


A carga cruzada de todas as variáveis observadas também foi maior do que as intercorrelações de todas as outras variáveis observadas para cada construto, conforme mostrado na Tabela 3. Assim, esses achados confirmam os critérios de avaliação de carga cruzada e fornecem uma validação aceitável para a validade discriminante do modelo de medição. Como resultado, o modelo conceitual proposto apoiou o fato de que o modelo de medição forneceu validação para a validade discriminante e validade convergente aceitável com confirmação de confiabilidade adequada.

Tabela 3. Cargas cruzadas de todas as variáveis observadas

\begin{tabular}{|c|c|c|c|c|c|c|c|}
\hline & ED & $\mathrm{EE}$ & IS & CF & $\mathrm{HA}$ & IC & CDU \\
\hline $\mathrm{ED} 1$ & 0,872 & 0,608 & 0,564 & 0,575 & 0,660 & 0,687 & 0,636 \\
\hline $\mathrm{ED} 2$ & 0,864 & 0,619 & 0,521 & 0,594 & 0,624 & 0,712 & 0,661 \\
\hline $\mathrm{ED}_{3}$ & 0,877 & 0,694 & 0,575 & 0,674 & 0,698 & 0,734 & 0,546 \\
\hline $\mathrm{ED} 4$ & 0,874 & 0,647 & 0,497 & 0,641 & 0,656 & 0,687 & 0,561 \\
\hline $\mathrm{ED}_{5}$ & 0,876 & 0,612 & 0,511 & 0,617 & 0,624 & 0,673 & 0,618 \\
\hline $\mathrm{EE} 1$ & 0,657 & 0,827 & 0,549 & 0,565 & 0,615 & 0,653 & 0,588 \\
\hline EE2 & 0,563 & 0,820 & 0,446 & 0,518 & 0,530 & 0,633 & 0,662 \\
\hline $\mathrm{EE}_{3}$ & 0,584 & 0,841 & 0,559 & 0,550 & 0,593 & 0,640 & 0,585 \\
\hline $\mathrm{EE}_{4}$ & 0,578 & 0,855 & 0,509 & 0,581 & 0,581 & 0,662 & 0,584 \\
\hline $\mathrm{EE}_{5}$ & 0,678 & 0,853 & 0,531 & 0,596 & 0,606 & 0,670 & 0,636 \\
\hline $\mathrm{IS}_{1}$ & 0,507 & 0,494 & 0,842 & 0,571 & 0,483 & 0,598 & 0,557 \\
\hline $\mathrm{IS}_{2}$ & 0,535 & 0,568 & 0,873 & 0,538 & 0,552 & 0,645 & 0,620 \\
\hline $\mathrm{IS}_{3}$ & 0,542 & 0,496 & 0,867 & 0,538 & 0,534 & 0,615 & 0,543 \\
\hline $\mathrm{IS}_{4}$ & 0,539 & 0,585 & 0,886 & 0,536 & 0,593 & 0,617 & 0,518 \\
\hline $\mathrm{CF}_{1}$ & 0,567 & 0,520 & 0,512 & 0,796 & 0,591 & 0,731 & 0,651 \\
\hline $\mathrm{CF}_{2}$ & 0,607 & 0,541 & 0,517 & 0,843 & 0,651 & 0,687 & 0,719 \\
\hline $\mathrm{CF}_{3}$ & 0,590 & 0,568 & 0,548 & 0,816 & 0,593 & 0,690 & 0,632 \\
\hline $\mathrm{CF}_{4}$ & 0.601 & 0,600 & 0,513 & 0,871 & 0,621 & 0,677 & 0,676 \\
\hline HA1 & 0,684 & 0,659 & 0,610 & 0,693 & 0,905 & 0,793 & 0,657 \\
\hline $\mathrm{HA} 2$ & 0,693 & 0,617 & 0,536 & 0,677 & 0,941 & 0,726 & 0,667 \\
\hline $\mathrm{HA}_{3}$ & 0,664 & 0,620 & 0,559 & 0,655 & 0,879 & 0,686 & 0,613 \\
\hline $\mathrm{HA}_{4}$ & 0,690 & 0,651 & 0,582 & 0,675 & 0,911 & 0,717 & 0,678 \\
\hline $\mathrm{HA}_{5}$ & 0,680 & 0,634 & 0,556 & 0,667 & 0,926 & 0,716 & 0,691 \\
\hline $\mathrm{IC}_{1}$ & 0,668 & 0,678 & 0,576 & 0,709 & 0,646 & 0,850 & 0,667 \\
\hline IC2 & 0,671 & 0,629 & 0,580 & 0,710 & 0,702 & 0,834 & 0,721 \\
\hline $\mathrm{IC}_{3}$ & 0,681 & 0,655 & 0,627 & 0,733 & 0,671 & 0,853 & 0,679 \\
\hline $\mathrm{IC}_{4}$ & 0,694 & 0,667 & 0,636 & 0,688 & 0,689 & 0,851 & 0,654 \\
\hline $\mathrm{CDU}_{1}$ & 0,521 & 0,533 & 0,487 & 0,683 & 0,586 & 0,647 & 0,779 \\
\hline $\mathrm{CDU}_{2}$ & 0,643 & 0,639 & 0,548 & 0,702 & 0,625 & 0,709 & 0,896 \\
\hline
\end{tabular}




\section{Modelo estrutural e teste das hipóteses}

Este estudo utilizou o método de PLS para testar se o valor dos coeficientes de caminho padronizados ( $\beta$ ) entre os vários construtos era estatisticamente significativo. Foi utilizado o coeficiente de determinação $R^{2}$ para medir o poder explicativo do modelo estrutural. Uma vez que o método de PLS não possui distribuição e não fornece o modelo de compatibilidade geral, para testar a significância do caminho estimado, foram utilizados o teste te a reamostragem por bootstrapping com geração de mil amostras a fim de explicar as relações entre as variáveis como teste das hipóteses e análise causal. Os coeficientes de caminho ( $\beta$ ) do modelo de equação estrutural, os valores do coeficiente de determinação $R^{2}$ e o valor da estatística t são mostrados na Figura 4 e na Tabela 4. Como previsto, os coeficientes de caminho ( $\beta$ ) no modelo hipotetizado e os valores-t correspondentes na Tabela 4 indicaram que a $\operatorname{ED}\left(\beta=0,355, \mathrm{f}^{2}=0,376, T=6,064, p=0,000\right)$, a $\operatorname{EE}\left(\beta=0,182, f^{2}=0,248, T=3,665, p=0,000\right)$, a IS $\left(\beta=0,167, f^{2}=0,267, T=3,513, p=0,000\right)$, as $C F\left(\beta=0,176, f^{2}=0,282, T=4,066, p=0,000\right)$ e o hábito $(\beta=0,318$, $f^{2}=0,245, T=3,492, p=0,001$ ) afetam positivamente a intenção comportamental de utilizar o Line Today. Os resultados confirmaram que $\mathrm{H}_{1}, \mathrm{H}_{2}, \mathrm{H}_{3}, \mathrm{H}_{4}$ e $\mathrm{H}_{5}$ foram apoiadas. A intenção comportamental afeta positivamente $\mathrm{O}$ comportamento de uso de utilizar o Line Today $\left(\beta=0,152, f^{2}=0,197, T=4,453, p=0,000\right)$, mostrando que $\mathrm{H} 6$ foi apoiada. As condições facilitadoras afetam positivamente o comportamento de uso de utilizar o Line Today ( $\beta=$ $\left.0,212, f^{2}=0,182, T=2,694, p=0,007\right)$, confirmando a hipótese H7. $\mathrm{O}$ hábito afeta positivamente o comportamento de uso de utilizar o Line Today $\left(\beta=0,242, \mathrm{f}^{2}=0,090, \mathrm{~T}=2,364, \mathrm{p}=0,018\right)$. Assim, a hipótese H8 foi suportada.

Tabela 4. Coeficiente de caminho, valor de $\beta, f_{2}$ de Cohen, e estatísticas $t$

\begin{tabular}{|c|c|c|c|c|c|}
\hline & Caminho hipotético & $\beta$ & $f^{2}$ & Estatística t & Valor-p \\
\hline $\mathrm{H}_{1}$ & $E D \rightarrow I C$ & 0,355 & 0,376 & 6,064 & 0,000 \\
\hline $\mathrm{H}_{2}$ & $\mathrm{EE} \rightarrow \mathrm{IC}$ & 0,182 & 0,248 & 3,665 & 0,000 \\
\hline $\mathrm{H}_{3}$ & $I S \rightarrow I C$ & 0,167 & 0,267 & 3,513 & 0,000 \\
\hline $\mathrm{H}_{4}$ & $C F \rightarrow I C$ & 0,176 & 0,282 & 4,066 & 0,000 \\
\hline $\mathrm{H}_{5}$ & $\mathrm{HA} \rightarrow \mathrm{IC}$ & 0,318 & 0,245 & 3,492 & 0,001 \\
\hline $\mathrm{H} 6$ & $\mathrm{IC} \rightarrow \mathrm{CDU}$ & 0,152 & 0,197 & 4,453 & 0,000 \\
\hline $\mathrm{H}_{7}$ & $\mathrm{CF} \rightarrow \mathrm{CDU}$ & 0,212 & 0,182 & 2,694 & 0,007 \\
\hline $\mathrm{H} 8$ & $\mathrm{HA} \rightarrow \mathrm{CDU}$ & 0,242 & 0,090 & 2,364 & 0,018 \\
\hline
\end{tabular}

$O$ coeficiente de determinação $R^{2}$ estimado explicou significativamente o modelo estrutural, com a ED, a EE, a IS, as CF e o hábito respondendo por 90,6\% da variância na intenção comportamental e por $52 \%$ da variância no comportamento de uso. Com base nos resultados, os valores de $\mathrm{R}^{2}$ foram considerados substanciais, e os achados da análise identificaram cinco fatores que aparecem como preditores da intenção comportamental e do comportamento de uso no modelo. 0 estudo também utiliza o $Q^{2}$ de Stone-Geisser para avaliar a relevância preditiva do modelo. $O Q^{2}$ no modelo do estudo é de o,698 e de 0,348, e, como sugerido por Chin (1998), um valor de $Q^{2}$ maior do que zero significa que o modelo tem capacidade preditiva. 
Figura 4. Modelo da equação estrutural

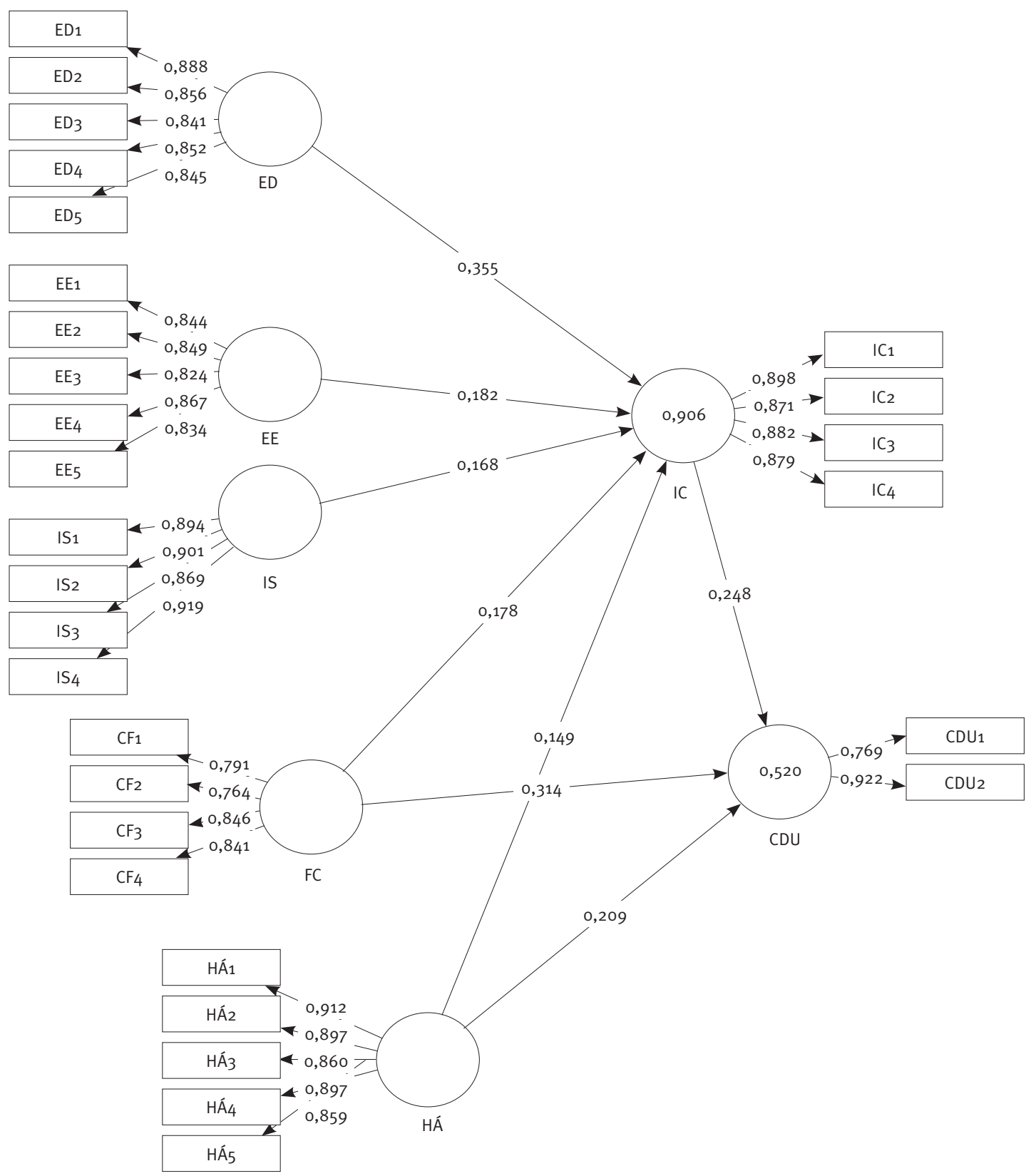


De acordo com a análise por PLS dos modelos de medição e do modelo estrutural, como esperado, ambos os modelos deram resultados estatisticamente significativos, e todas as hipóteses foram aceitas. Embora o valor de $\beta$ não seja elevado, todos são significativos, e o poder explicativo do $R^{2}$ chega a $52 \%$ e $90 \%$, assim a distribuição do valor de $\beta$ é estatisticamente significativa. Na Tabela 4, pode-se ver que o tamanho do $\beta$ indica o grau de influência da variável independente, e seu impacto é mais alto sobre a ED, seguida pelo hábito, e assim por diante. Os resultados deste estudo confirmaram os fatores que afetam a intenção comportamental, e podem apoiar o poder explicativo quanto ao comportamento do usuário de utilizar novas tecnologias.

\section{CONCLUSÃO}

Este estudo concentra-se no ponto de vista do usuário, e utiliza a Utaut2 como base teórica para explorar o comportamento de uso do Line Today. Com base nos resultados empíricos, todos os fatores exercem um impacto positivo sobre as intenções comportamentais. Quando o usuário acredita que o Line Today pode satisfazer seu desejo de assistir a notícias, cresce sua intenção de utilizá-lo. De acordo com os resultados, percebe-se que, se os usuários consideram o Line Today prático e útil para suas vidas, isso aumenta sua disposição de usá-lo. Quando os usuários consideram que a interface de usuário do Line Today é fácil de operar e não requer muito esforço, eles ficam mais dispostos a utilizá-lo.

Entretanto, quando o usuário utiliza o Line Today, ele o faz devido às opiniões de outras pessoas importantes para ele. Esse resultado é consistente com pesquisas anteriores (Venkatesh et al., 2003, 2012). Quanto mais os usuários sentem que seus parentes e amigos influenciam seu uso do Line Today, mais sua intenção de utilizá-lo aumenta. Além disso, quando os usuários pensam que as tecnologias de software e hardware e recursos de equipamentos atualmente disponíveis podem apoiar o grau de uso pessoal do Line Today, e quanto mais os usuários sentem um grau de apoio ambiental ao utilizar a plataforma, mais eles a utilizarão.

Venkatesh et al. (2012) acreditam que o hábito seja a tendência a utilizar a tecnologia automaticamente. Foi apontado, em estudos anteriores, que o hábito é um ato anterior e uma ideia-chave que move o comportamento repetitivo. Quando os usuários utilizam o Line Today como sua plataforma de escolha para assistir a notícias, isso aumenta sua intenção de utilizá-lo. A literatura sobre os modelos Utaut e Utautz também apontou que a intenção comportamental exerce um impacto significativo sobre o uso efetivo de uma tecnologia, independentemente de a tecnologia ser utilizada no ambiente organizacional ou no ambiente do consumidor (Venkatesh et al., 2003, 2012). No entanto, embora as condições facilitadoras deste estudo possuam efeitos positivos sobre $o$ comportamento de uso, alguns dos resultados não tiveram um impacto significativamente positivo, consistente com os resultados de Weng e Huang (2017) sobre o comportamento de uso do Line TV. Infere-se que, embora dispor de recursos e conhecimento suficientes para utilizar o Line Today possa aumentar a disposição de utilizá-lo, isso pode não aumentar o tempo e a frequência de uso efetivos, o que pode estar relacionado ao fato de que o usuário ainda dispõe de outras opções.

\section{Contribuições do estudo}

O estudo visa principalmente pesquisar a intenção comportamental e o comportamento de uso dos usuários do Line Today na plataforma móvel de notícias. Atualmente, há poucas pesquisas sobre o Line Today. Portanto, o 
estudo utiliza a Utaut2 para analisar os fatores que afetam as intenções comportamentais e os comportamentos de uso do Line Today. Embora já se tenha provado empiricamente que a Utautz é um modelo cientificamente aceito, relativamente bem-estruturado, bem discutido e adequado a pesquisas sobre o comportamento do consumidor, como foi proposto há apenas alguns anos, as pesquisas utilizando esse modelo não são tão extensas quanto as que utilizam a TAM. As contribuições específicas desta pesquisa são as seguintes: (1) ela confirma que a Utaut2 pode ser utilizada para verificar as intenções de comportamento e o comportamento de uso dos usuários do Line Today, possuindo bom poder explicativo; (2) estudos têm mostrado que a ED, a EE, a IS, as CF e o hábito exercem um impacto significativo sobre a intenção comportamental, enquanto a intenção comportamental e o hábito exercem um impacto positivo sobre o comportamento de uso (os resultados deste estudo estão disponíveis para estudiosos que, no futuro, desejem conduzir pesquisas relevantes sobre o tema); (3) 0 estudo concluiu que os usuários do Line Today são majoritariamente jovens com idades entre 21 e 30 anos, principalmente estudantes universitários. O Line Today possui um grande número de usuários na comunidade Line. Fica claro que, no ambiente desenvolvido da internet, a base de usuários do software de comunidade original cresce a uma taxa maior do que a de outros aplicativos de notícias, e, ao adquirir novos conhecimentos por meio da plataforma, os jovens aceitam a nova plataforma de TI de modo relativamente mais fácil.

\section{Implicações práticas do estudo}

Com base no processo e nos resultados da pesquisa, o estudo sugere implicações gerenciais e práticas para gestores na área de estratégia. Em termos dos serviços oferecidos pelo Line Today, os resultados sobre ED confirmam que a plataforma permite aos usuários realizar seu objetivo de assistir a notícias. Os resultados do estudo também sugerem que o Line Today é útil e eficiente na vida, possibilitando que as pessoas acessem conhecimentos e as informações mais recentes instantaneamente; Além disso, pessoas relativamente jovens aceitam a nova plataforma de TI mais rapidamente. No que tange à EE, os gestores podem aprimorar o design e a operação da interface da plataforma de modo a torná-la mais fácil para que os usuários iniciem seu uso. Com relação ao impacto da IS, como o Line Today ainda é uma plataforma de conteúdo, há menos envolvimento na interação dos usuários ao assistir a notícias. Atualmente, eles podem apenas postar seus próprios comentários abaixo dos conteúdos de notícias e exibi-los na tela em que estão assistindo. No futuro, poderão aproveitar o conteúdo do Line Today nas salas de bate-papo do Line, em eventos ao vivo e em janelas de bate-papo, aumentado o grau de interação. Além do conteúdo diversificado e conciso, gestores não podem ignorar o fator da IS, pois ele também pode aumentar as intenções comportamentais. Assim, espera-se que os gestores possam continuar a aprimorar as vantagens existentes, integrar diferentes plataformas e eliminar suas falhas, a fim de aumentar a intenção de uso dos usuários.

A plataforma móvel de notícias do Line Today também pode introduzir serviços diferenciados para diferentes grupos de usuários, lançar conteúdo que pode ser de interesse, introduzir preferências com base nas características dos diferentes grupos étnicos ou oferecer mais níveis de serviço, de modo a atender a diferentes tipos de usuários. Ela pode também buscar personalizar o serviço com horários e leituras diferentes. Por outro lado, deve ser capaz de combinar serviços relacionados, além de notícias, tais como recomendações de bares, restaurantes e lojas, notícias e informações sobre produtos, aliados à conveniência dos dispositivos móveis, com um marketing que atinja mais profundamente a vida do usuário. Por exemplo, em termos de entretenimento, pode oferecer outros tipos de conteúdo de notícias, relacionados, por exemplo, a viagens, moda e astrologia, e utilizar o com- 
partilhamento em comunidades para satisfazer a disposição dos usuários de compartilhar, aumentando, assim, as intenções de uso. Novas opções de entretenimento podem ser combinadas com a plataforma Line existente, de modo a desenvolver outras de modo a desenvolver novas funções, tais como competições ao vivo, jogos de basquete combinados com notícias on-line, a fim de estimular a curiosidade desse grupo e aumentar suas intenções de uso. Além disso, a plataforma pode também aumentar seu conteúdo de áudio e vídeo, especialmente em notícias, entretenimento e estilo de vida, e aumentar o conteúdo ao vivo, incluindo shows, cerimônias de premiação e eventos esportivos.

\section{Limitações e direcionamentos futuros}

Embora o estabelecimento da estrutura da pesquisa, a elaboração do questionário e a coleta e análise dos dados tenham sido rigorosos e objetivos, o processo da pesquisa foi inevitavelmente limitado, devido a restrições de tempo, recursos e pessoal. Apresentam-se, a seguir, as restrições da pesquisa e recomendações a pesquisadores para estudos futuros. Sob restrições de tempo e financiamento, a utilização de um questionário impresso e de questionários on-line para a coleta de dados, bem como a amostragem por conveniência, apresenta limitações em termos da representatividade da amostra neste estudo. A amostra foi composta por indivíduos com idades entre 21 e 30 anos, a maioria dos quais estudantes, portanto a discussão sobre as características gerais dos usuários é limitada. No futuro, estudos relevantes podem ser ampliados no sentido de incluir Taiwan inteira, aumentando as dimensões da amostra, a fim de expandir os resultados da pesquisa e aumentar sua validade externa. Como a amostra do estudo não é grande, mas há cinco variáveis dependentes que podem resultar em um valor de $\beta$ mais baixo, recomenda-se, no futuro, aumentar o tamanho da amostra, a fim de melhorar o valor preditivo do $\beta$. No entanto, as características deste estudo são consistentes com as características do principal grupo étnico de Taiwan, que é composto, em sua maioria, por jovens, assim os achados da pesquisa ainda são valiosos. A análise quantitativa deste estudo não avalia os pensamentos dos sujeitos pesquisados, mas apenas a tendência da situação geral do grupo, portanto esse fato pode afetar seu efeito moderador, devido a pequenas diferenças.

Atualmente, na plataforma do Line Today, os usuários podem analisar suas informações de navegação, o que lhes permite visualizar suas listas de favoritos e compartilhar conteúdo de notícias de modo instantâneo. Recomenda-se oferecer aos usuários serviços de interligação de domínios, tais como notícias sobre o tráfego de trens durante o Festival da Primavera. Isso pode ser feito por meio de links para reservas de passagens, exibição de notícias relacionadas a viagens e informações relevantes (acomodação, preços de passagens aéreas etc.). Com os recursos existentes, recomenda-se aprimorar a apresentação do conteúdo de notícias na plataforma do Line Today com a adição de funções personalizadas que integrem a localização das pessoas, de modo a oferecer notícias mais relevantes sobre o local onde se encontrem. A plataforma pode analisar os tipos de notícias em que as pessoas navegam e recomendar notícias e informações relevantes. Pode também oferecer informações aprofundadas ao público sobre o conteúdo das notícias, a fim de aumentar seu envolvimento com as elas ou melhorar sua conexão com a vida.

\section{NOTA DE TRADUÇÃO}

Nota de tradução: as abreviações referentes às teorias foram mantidas em inglês para fins de padronização. 


\section{REFERÊNCIAS}

Alalwan, A. A. (2020). Mobile food ordering apps: An empirical study of the factors affecting customer e-satisfaction and continued intention to reuse. International Journal of Information Management, 50, 28-44. doi: 10.1016/j. ijinfomgt.2019.04.008

Ali, F., Nair, P. K., \& Hussain, K. (2016). An assessment of students' acceptance and usage of computer supported collaborative classrooms in hospitality and tourism schools. Journal of Hospitality, Leisure, Sport \& Tourism Education, 19, 51-60. doi: 10.1016/j.jhlste.2016.03.002

Appel, G., Grewal, L., Hadi, R., \& Stephen, A. (2019). The future of social media in marketing, Journal of the Academy of Marketing Science, 48(1), 1-17. doi: 10.1007/S11747-01900695-1

Baabdullah, A. M. (2018). Consumer adoption of mobile social network games (M-SNGs) in Saudi Arabia: The role of social influence, hedonic motivation and trust. Technology in Society, 53, 91-102. doi: 10.1016/j.techsoc.2018.01.004

Business Next. (2016). 17 million Taiwanese are using it! Three graphs to see the user analysis of Line. Recuperado de https:// www.bnext.com.tw/article/41433/line-user in-taiwan-is more-than-90-percent

Business Next. (2018). Line Taiwan's monthly active users exceeded 21 million, and the three major functions and usage rate ranked first in the world. Recuperado de https://www. bnext.com.tw/article/51783/line-linetoday-linestore-sticker

Cao, Q., \& Niu. X. (2019). Integrating context-awareness and Utaut to explain Alipay user adoption. International Journal of Industrial Ergonomics, 69, 9-13. doi: 10.1016/j. ergon.2018.09.004

Chao, C. M. (2019). Factors determining the behavioural intention to use mobile learning: An application and extension of the Utaut model. Frontiers in Psychology, 10(1), 1-14. doi: $10.3389 /$ fpsyg.2019.01652

Chin, W. W. (1998). The partial least squares approach for structural equation modeling. In G. A. Marcoulides (Ed.), Modern methods for business research (pp. 295-236). London, UK: Lawrence Erlbaum Associates.

China Times. (2015). Media review-social media changed the news. Recuperado de https://www.chinatimes.com/ newspapers/20150728000503-260109?chdtv

China Times. (2017). Line launches new service-online and offline zero distance. Recuperado de https://www.chinatimes.com/ newspapers/20171002000078-260204?chdtv

China Times (2017b). LINE's users have more than 18 million annual events opened by her. Retrieved from http://www. chinatimes.com/realtimenews/20170310004183-260412

Chopdar, P. K., Korfiatis, N., Sivakumar, V. J., \& Lytras, M. D. (2018). Mobile shopping apps adoption and perceived risks: A cross-country perspective utilizing the Unified Theory of Acceptance and Use of Technology. Computers in Human Behavior, 86, 109-128. doi: 10.1016/j.chb.2018.04.017
Davis, F. D. (1989). Perceived usefulness, perceived ease of use, and user acceptance of information technology. MIS quarterly, 13(3), 319-340. doi: 10.2307/249008

Digital News Report. (2018). Recuperado de http://media. digitalnewsreport.org/wp-content/uploads/2018/06/digitalnews-report-2018.pdf

Dwivedi, Y. K., Rana, N. P., Jeyaraj, A., Clement, M., \& Williams, M. D. (2019). Re-examining the unified theory of acceptance and use of technology (Utaut): Towards a revised theoretical model. Information Systems Frontiers, 21(3), 719-734. doi: 10.1007/s10796-017-9774-y

ETtoday. (2018). Taiwan internet media ranked first in ETtoday! Recuperado de https://www.ettoday.net/ news/20180622/1196359.htm

Fishbein, M. A., \& Ajzen, I. (1975). Belief, attitude, intention and behaviour: An introduction to theory and research. Reading,USA: Addison Wesley.

Global Views. (2018). Line Today to create a content portal to see the news, life size. Recuperado de https://www.gvm.com.tw/ article.html?id $=44828$

Hallikainena, H., Alamäki, A., \& Laukkanen, T. (2019). Lead users of business mobile services. International Journal of Information Management, 47(1), 283-292. doi: 10.1016/j. ijinfomgt.2018.10.018

Hair, J. F., Anderson, R. E., Tatham, R. L., \& Black, W. C. (1998). Multivariate Data Analysis (5th ed.). Upper Saddle River, NJ: Prentice Hall.

Harris, R. J. (1985). A primer of multivariate statistics (2nd ed.). New York, USA: Academic Press.

Herrero, A., Martín, H. S., \& Salmones, M. G. (2017). Explaining the adoption of social networks sites for sharing usergenerated content: A revision of the Utaut2. Computers in Human Behavior, 71, 209-217. doi: 10.1016/j.chb.2017.02.007

Lee, S. K., Lindsey, N. J., \& Kim, H. S. (2017). The effects of news consumption via social media and news information overload on perceptions of journalistic norms and practices. Computers in Human Behavior, 75(1), 254-263. doi: 10.1016/j. chb.2017.05.007

Line Today. (2017). LINE Today new upgrade. Recuperado de https://linecorp.com/zh-hant/pr/news/zh-hant/2017/1663

Market Intelligence \& Consulting Institute. (2016). Mobile app consumer survey-super love chat more than $80 \%$ use social communication app every day. Recuperado de https:// mic.iii.org.tw/IndustryObservations_PressReleaseo2. aspx?sqno $=423$

Meel, P., \& Vishwakarma, D. K. (2019). Fake news, rumour, information pollution in social media and web: A contemporary survey of state-of-the-arts, challenges and opportunities. Expert Systems with Applications, 153(1), 1-26. doi: 10.1016/j.eswa.2019.112986 
Nunnally, J. C. (1978). Psychometric theory. New York, USA: McGraw-Hill.

Ramírez-Correa, P., Rondán-Cataluña, F. J., Arenas-Gaitán, J., \& Martín-Velicia, F. (2019). Analysing the acceptation of online games in mobile devices: An application of Utaut2. Journal of Retailing and Consumer Services, 50, 85-93. doi: 10.1016/j. jretconser.2019.04.018

Shiferaw, K. B., \& Mehari, E. A. (2019). Modeling predictors of acceptance and use of electronic medical record system in a resource limited setting: Using modified Utaut model. Informatics in Medicine Unlocked, 17(1), 1-9. doi: 10.1016/j.imu.2019.100182

Tamilmani, K., Rana, N. P., Prakasam, N., \& Dwivedi, Y. K. (2019, June). The battle of brain vs. heart: A literature review and meta-analysis of "hedonic motivation" use in UTAUT2. International Journal of Information Management, 46, 222235. doi: 10.1016/j.ijinfomgt.2019.01.008
Tavis. (2012). Top ten trends in 2012: Social media redefines news communication model. Recuperado de http://tavis.tw/ files/13-1000-12562.php

Venkatesh, V., Morris, M. G., Davis, G. B., \& Davis, F. D. (2003). User acceptance of information technology: Toward a unified view. MIS quarterly, 27(3), 425-478. doi: 10.2307/30036540

Venkatesh, V., Thong, J. Y., \& Xu, X. (2012). Consumer acceptance and use of information technology: Extending the unified theory of acceptance and use of technology. MIS Quarterly, 36(1), 157-178. doi: 10.2307/41410412

Weng, C., \& Huang, H (2017). A study on the use behaviour of Line TV based on the Utaut2 and digital lifestyle scale. Journal of Information Society, 33, 17-63. doi: 10.29843\%2fJCCIS.201707_ (33).0002

\section{CONTRIBUIÇÕES DOS AUTORES}

A conceitualização e a abordagem teórico-metodológica foram coordenadas por Lisa Y. Chen. A revisão teórica foi conduzida por Lisa Y. Chen e Yi-Jhen Chen. A coleta de dados foi realizada por Yi-Jhen Chen. A análise dos dados, a redação e a revisão final foram realizadas por Lisa Y. Chen.

\section{ERRATA}

Onde se lia: Kaohsiung Polytechnic Institute

Leia-se: I-Shou University 